\title{
Élaborer une stratégie de recherche
}

Developing a Research Strategy

Karine Thilleul

\section{(2) OpenEdition}

Journals

Édition électronique

URL : http://journals.openedition.org/crau/537

DOI : $10.4000 /$ crau. 537

ISSN : 2547-5746

\section{Éditeur}

Éditions du patrimoine

\section{Édition imprimée}

Date de publication : 1 novembre 2012

Pagination : 40-49

ISBN : 978-2-7577-0108-9

ISSN : 1296-4077

Référence électronique

Karine Thilleul, «Élaborer une stratégie de recherche », Les Cahiers de la recherche architecturale et urbaine [En ligne], 26/27 | 2012, mis en ligne le 01 novembre 2017, consulté le 19 avril 2019. URL http://journals.openedition.org/crau/537 ; DOI : 10.4000/crau.537 
Dans quelle mesure les études d'architecture apportent-elles les outils nécessaires à un travail de recherche ? Comment définir une problématique d'ensemble, notamment face à la diversité des sources? Quelles approches et stratégies de recherche mettre en place?

\section{Élaborer une stratégie
de recherche}

KARINE THILLEUL

Dans quelle mesure les études d'architecture apportent-elles les outils nécessaires à un travail de recherche ? La possibilité, existant théoriquement depuis 2005, d'entreprendre une thèse de doctorat en architecture sans passer par un cursus de master spécialisé dans la recherche amène à examiner cette question ${ }^{1}$.

La formation d'architecte est assez généraliste et comporte des champs très divers. L'ensemble des cours théoriques et pratiques que doit suivre l'étudiant permet d'acquérir un bagage culturel relativement conséquent, notamment au travers des différents enseignements liés aux sciences humaines et sociales; les exercices de projet, par le biais de la recherche de références, représentent d'autres opportunités d'acquérir des connaissances, lesquelles sont approfondies par des exercices pratiques liés à la maîtrise de la conception architecturale.

Cependant, les méthodologies liées à la recherche et aux approches historiques ne sont que très peu abordées au cours des études d'architecture. Un cursus approfondi en histoire de l'art apporte certainement

1. Créé par le décret $n^{\circ} 2005-734$

du 30 juin 2005 relatif aux études d'architecture (NOR: MCCB0500411D), le doctorat en architecture permet de situer les études d'architecture dans le cadre européen de la réforme LMD. 
des bases plus solides dans les domaines de la méthodologie et de l'écriture. C'est par la pratique que l'étudiant en architecture ou le jeune architecte qui s'essaiera à la recherche parviendra à acquérir les outils nécessaires et à mettre en place une stratégie de recherche. II dépend, à ce niveau, de différentes contingences relationnelles liées à son cursus.

Toutefois, la maîtrise du domaine de la conception architecturale peut représenter un modèle de réflexion auquel se référer. Il existe des analogies entre l'exercice du projet architectural et celui du travail de recherche rédigé : tous deux nécessitent une quête de la plus grande cohérence possible, et ce, à différents niveaux. Ainsi, un projet architectural de qualité passe nécessairement par une réflexion sur l'homogénéité des différents éléments qui le constituent, depuis le plan masse jusqu'au dessin des détails; de la même manière, mener une recherche scientifique sur un sujet donné nécessite la définition d'un corpus, d'un plan et d'une charte de rédaction cohérents. Dans chacune de ces situations, une partie des règles doit être déterminée par l'auteur : c'est là que réside une grande partie de la difficulté de ces exercices. II existe bien entendu des règles externes, fixées par les textes législatifs en premier lieu, puis par le contexte local. Mais, dans les deux cas, c'est à l'architecte ou au chercheur qu'il appartient de définir une partie des règles du jeu auxquelles il devra se conformer le plus fidèlement possible afin de viser à la production d'un raisonnement porteur de sens. S'il choisit de modifier I'une des règles en cours de route, il lui faudra vérifier que l'ensemble du travail produit dès le départ s'y conforme, et, le cas échant, le modifier afin de pallier toute divergence.

Tout comme l'architecte peut choisir de commencer son travail par un schéma d'ensemble, par une vue perspective d'un lieu qui lui semble particulièrement intéressant, ou encore par une réflexion sur un détail, afin de mettre en place une première accroche dans le processus créatif et de commencer à établir des règles, le chercheur a différentes possibilités pour entamer son travail : il pourra partir d'une situation concrète particulière qui interroge ses capacités réflexives, d'une intuition théorique, d'un schéma chronologique... L'étude du contexte historique global relatif à une situation donnée permettra d'en enrichir l'analyse et vice versa; ainsi, pour le chercheur comme pour l'architecte, quel que soit le point de départ du travail, il est nécessaire d'effectuer des allers et retours entre les différentes échelles, globales et locales, d'établir différents parti pris et de les respecter.

\section{Approches stratégiques du projet de recherche}

Les formes que prennent les travaux de recherche en histoire de l'architecture sont souvent influencées par les modèles, issus de l'histoire de l'art, de la monographie et de la biographie. Une approche thématique, liée à une problématique ou à une typologie donnée, permet de les combiner.

Dans le cas de ma thèse, j'ai fréquemment navigué entre objets et sujets, familles d'édifices et familles de concepteurs. La problématique centrale qui a guidé le début de mon travail était basée sur l'analyse des 
relations entre architectes et grand public, sur les causes des difficultés de communication qu'ont connues et que connaissent toujours ces relations, et sur l'étude des médiations culturelles ayant contribué à établir des passerelles. Ce thème se trouvait donc situé aux confluents interdisciplinaires de l'architecture, de I'histoire et de la sociologie.

Cette idée de problématique, mise en place au début du master recherche en 2005-2006, portait en filigrane la trace d'un mémoire réalisé l'année précédente. II s'agissait d'une première réflexion sur les liens entre image et espace. J'avais commencé à étudier la médiatisation des bâtiments : comment les photographies permettent de véhiculer leur image partout dans le monde, et comment le choix des points de vue et la présentation des images peuvent influencer très fortement la représentation mentale que l'on se fait des édifices, allant parfois même jusqu'à substituer une vision idéalisée, souvent chargée de symboles, aux espaces réels ${ }^{2}$.

La problématique que je cherchais alors à définir se situait aux confluents de ces deux thèmes. Cependant, dans le cadre d'un master recherche, il est rapidement apparu que les méthodes de recherche historique avaient besoin d'objets concrets sur lesquels se porter pour produire le genre de résultats attendus : des connaissances nouvelles. La nécessité de choisir un objet symbolique représentatif des relations entre architectes et grand public s'est imposée, et la maison individuelle, qui, plus que tout autre programme, représente le lieu de cristallisation des pratiques et des attentes les plus personnelles des spécialistes comme de celles du grand public, a été retenue.
La maison industrialisée métallique, objet d'utopies d'architectes mal-aimé du grand public, a été pointée en particulier. II s'est ensuite avéré nécessaire de rebondir sur une problématique plus spécifique à cette catégorie d'objets, qui a été définie de la manière suivante : quelles sont les stratégies commerciales choisies par les concepteurs d'avant-garde pour vendre des produits innovants? Quels médias utilisent-ils ? Dans quelle mesure les moyens de communication utilisés, images et langages, influencent-ils leur réception par le grand public ? À cette problématique, définie de manière plus précise, a ensuite correspondu le choix d'un corpus plus spécifique.

\section{Objets de recherche matériels et immatériels \\ Fils conducteurs et lignes directrices}

Les maisons ont joué, pour la définition du sujet, le rôle d'objets intermédiaires entre plusieurs problématiques, en se présentant comme des objets tangibles permettant d'interroger les champs de recherche plus intangibles, voire abstraits, que représentaient les médiations entre architectes et grand public.

Pour la mise en place du plan de thèse, il a été nécessaire de délimiter un domaine d'étude précis, ce qui a impliqué d'autres stratégies. C'est à ce moment-là que la constitution d'un corpus rassemblant plusieurs concepteurs, certains célèbres et d'autres relativement inconnus, tous liés par leur volonté de produire des maisons industrialisées métalliques, a débuté.

La région Lorraine, marquée par les destructions des deux guerres mondiales, et par ailleurs forte d'une

2. Les travaux de Walter Benjamin (L'œuvre

d'art à l'époque de sa reproductibilité technique, version de 1935, extrait de Fuvres, t. 3, Paris, Gallimard, 2000), de Beatriz Colomina (La publicité du privé, de Loos à Le Corbusier, Orléans, Éditions HYX, 1998), et de Rosalind Krauss (Le photographique, pour une théorie des écarts, Paris, Macula, 1991) ont constitué les principales références de cette recherche. 
histoire industrielle singulière liée à l'industrie sidérurgique, se présentait comme un lieu propice aux investigations expérimentales autour de l'habitat industrialisé. En effet, les destructions liées aux deux guerres, la nécessité de bâtir rapidement des maisons bon marché, dans le cadre d'habitats transitoires ou de cités ouvrières, offrent un contexte historique particulièrement riche. C'est sur l'un des plus célèbres constructeurs de maisons en série, Jean Prouvé, que se sont d'abord portées les recherches, et en particulier sur les maisons en série conçues durant la Reconstruction d'après la Seconde Guerre mondiale (ill. 1).

\section{Maison Métropole}

exposée à Roubaix.

Photographie

\section{$11 \times 17 \mathrm{~cm}$.}

Institut pour l'histoire

de l'aluminium

coll. photographique

de l'Aluminium Français,

album 141, image A5017.

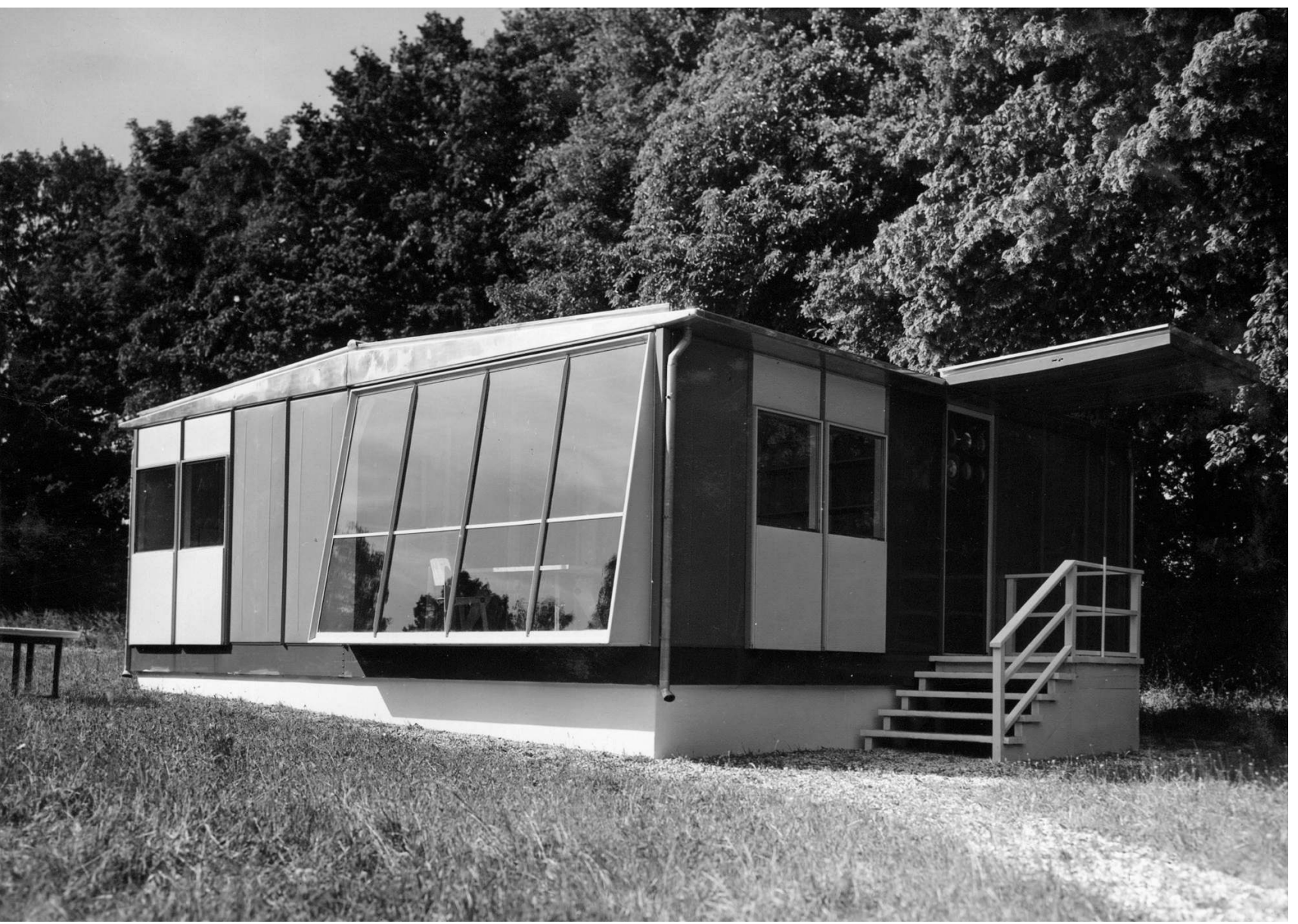


Le début des années 1950 représentait en effet pour la problématique un intérêt particulier, la Reconstruction donnant lieu à toutes sortes de structures de communication, et des événements comme le Salon des Arts Ménagers, existant déjà avant-guerre, devenant d'importantes occasions de rencontre entre architectes et grand public, sur l'impulsion, notamment, du ministère de la Reconstruction et de l'urbanisme.

La recherche a donc porté, dans un premier temps, sur les maisons en aluminium présentées par les deux frères Prouvé, Henri, architecte, et Jean, constructeur au Salon des Arts Ménagers : la maison à portique (1950) et la maison à coque $(1951)^{3}$. À travers ces deux cas particuliers, la question des structures de communication était abordée d'une manière plus générale, et interrogée comme l'une des causes possibles de l'échec de la production en série des maisons envisagées par Jean Prouvé 4 .

La découverte du travail d'un autre constructeur, Ferdinand Fillod, qui a produit de nombreuses maisons préfabriquées dans l'entre-deux guerres, a été une autre étape importante de la recherche. Elle a permis d'aborder la question de la production de maisons industrialisées en Lorraine dans les années 1920-1930, lesquelles se sont rapidement révélées comme une période clé qui voit apparaître les toutes premières grandes expériences de sérialisation de maisons individuelles, facilitées par le contexte législatif, et en particulier par la loi Loucheur (1928). Les expériences concernant les maisons industrialisées métalliques restent, à cette époque, marginales : seuls quelques architectes et constructeurs d'avant- garde s'essaient à cet exercice qui deviendra bien plus courant avec la Reconstruction d'après la Seconde Guerre mondiale. Parmi eux, Ferdinand Fillod n'a laissé que peu de traces dans l'historiographie des maisons préfabriquées, et son nom reste relativement inconnu ; cependant, sa démarche originale et sa production prolifique sont dignes d'intérêt. Jurassien d'origine, il a reçu, à l'instar de Jean Prouvé, une formation d'artisan; il ouvre un atelier de chaudronnier-soudeur à Saint-Amour, dans le Jura, en 1921. Son entreprise connaît une croissance rapide, et devient en quelques années I'usine du Grand SaintMichel, manufacture de tôlerie Fillod à Saint-Amour ${ }^{5}$ (ill. 2). Constructeur innovant, Ferdinand Fillod ne se contente pas de cette économie de fonctionnement, et procède à de nombreuses recherches concernant la construction préfabriquée en acier, et conçoit notamment des systèmes économiques, rapides et efficaces de production de maisons en série, mis en valeur dans différents documents publicitaires (ill. 3). Il met au point en 1928 un procédé de préfabrication du bâtiment qu'il fait breveter, et avec lequel seront réalisés de nombreux bâtiments, écoles, hôpitaux, casernes, cités ouvrières, immeubles et bâtiments publics entièrement en acier, en France et à l'étranger. Il choisit en 1929 de s'installer en Moselle, afin de se rapprocher des usines sidérurgiques, s'associe aux maîtres de forge De Wendel, et fonde la société de Constructions métalliques Fillod ( $\mathrm{CMF}$ ), basée à Hayange et Florange. Son usine de Florange, qui emploie jusqu'à 900 salariés, produit des milliers de mètres carrés de bâtiments préfabriqués ${ }^{6}$.
3. «La Maison Prouvé, première maison française entièrement préfabriquée », Le Décor d'aujourd'hui, n 55, 1950 ; « La septième exposition de l'habitation ", L'Architecture d'aujourd'hui, $n^{\circ} 28$, février 1950 ; «Une nouvelle maison préfabriquée au Salon des Arts Ménagers », Techniques et Architecture, n 3-4, 1951 ; " Huitième exposition de l'habitation, habitation type, Henri Prouvé architecte", L'Architecture d'aujourd'hui, $n^{\circ}$ 34, février-mars 1951 p. 6-15; «L'élément coque de Jean
Prouvé », Le Décor d'aujourd'hui, n 62, 1951, p. 178-179.

4. Voir à ce sujet, Axel Venacque, «Un patrimoine prototype ", dans Catherine Dumont d'Ayot et Bruno Reichlin (dir.), Jean Prouvé, la poétique de l'objet technique, Weil am Rhein, Vitra Design Museum, 2006, p. 356-361.
5. Florence Amiaux, L'industrialisation selon Ferdinand Fillod, éditorial Bertrand Lemoine, catalogue de l'exposition réalisée à la demande d'Arcelor par le CAUE de la Moselle, Metz, caue de la Moselle, 2002, p. 5.

6. Ibid. 


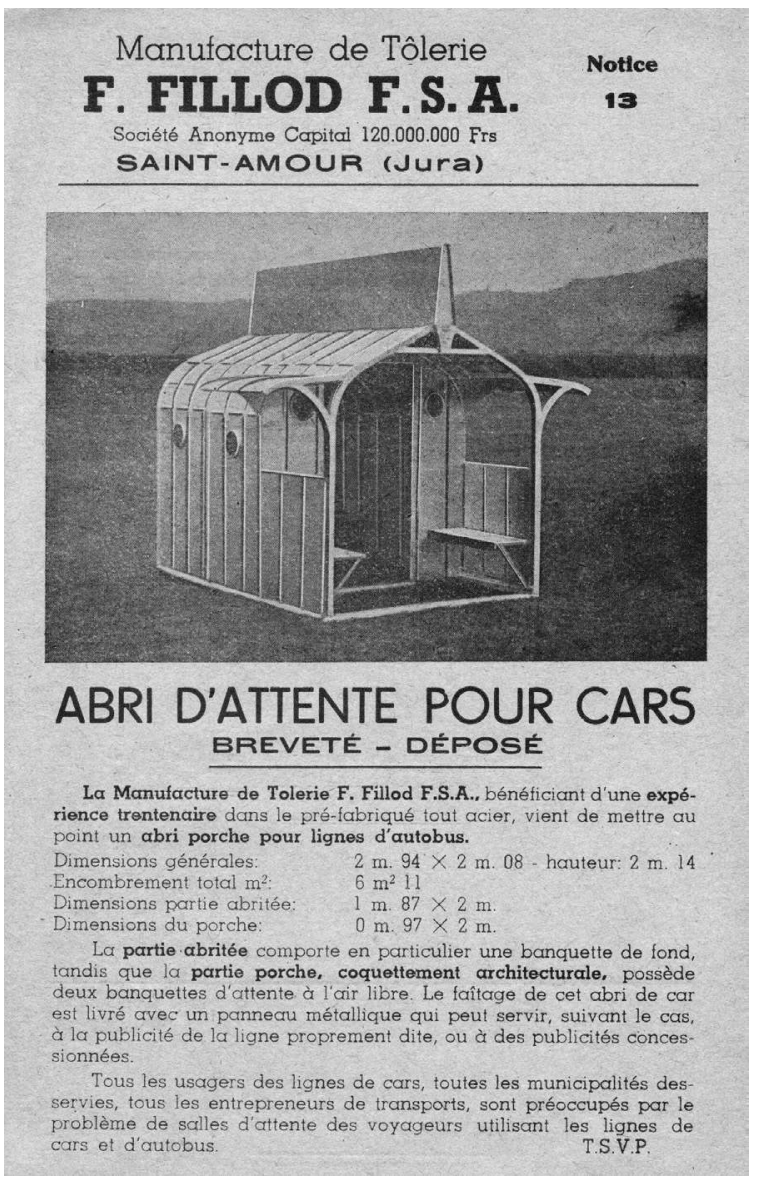

Notice « Abri

d'attente pour cars ",

Manufacture de

tôlerie F. Fillod F.S.A.

à Saint-Amour (Jura),

années 1950.

Coll. particulière

Gilles Rudelle.

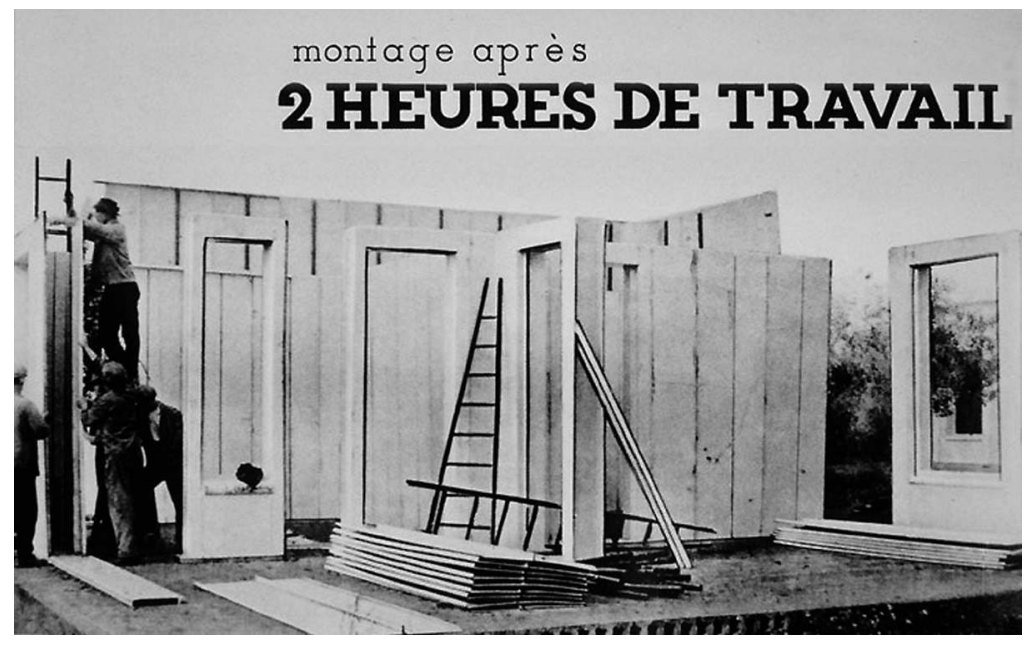

Document publicitaire pour les maisons en acier de Ferdinand Fillod, "Montage après deux heures de travail ", non daté. Coll. particulière Gilles Rudelle. 
Par la suite, après l'étude des conditions de production et de commercialisation des maisons industrialisées durant l'entre-deux guerres et la Reconstruction des années 1950, une troisième période clé a été définie, celle des Trente Glorieuses. Une trame historique chronologique s'est mise en place, dessinant une histoire des constructeurs de maisons industrialisées métalliques en Lorraine, depuis les pionniers jusqu'aux héritiers ; un bornage chronologique s'est ainsi ajouté au bornage géographique précédemment envisagé.

Le troisième concepteur étudié pour la période des Trente Glorieuses est Robert Anxionnat qui, au cours de sa carrière, a été l'auteur de plusieurs projets de maisons préfabriquées, lesquelles ont fait l'objet de diverses démarches promotionnelles ${ }^{7}$. Cette production, qui pourrait sembler en marge de l'activité d'un architecte plus connu pour ses projets d'architecture intérieure de bâtiments publics ${ }^{8}$ a cependant été le lieu $d^{\prime}$ un grand investissement personnel, lisible tant dans la qualité spatiale des maisons que dans le soin minutieux apporté à leurs détails constructifs.

C'est en 1968, année où l'agence de Robert Anxionnat est en pleine expansion, avec une nouvelle succursale à Paris et avec des succès engrangés à I'occasion de plusieurs concours importants, que l'architecte commence à s'intéresser aux maisons industrialisées. Deux premiers prototypes sont présentés lors de l'exposition Eurodesign'à Nancy en 1969 : la maison MAM à structure métallique ${ }^{10}$ (ill. 4) et la maison en béton (ill. 5) qui ne sera pas développée par la suite.

La maison MAM occupe une place à part dans la production d'Anxionnat. Conçue hors commande, elle

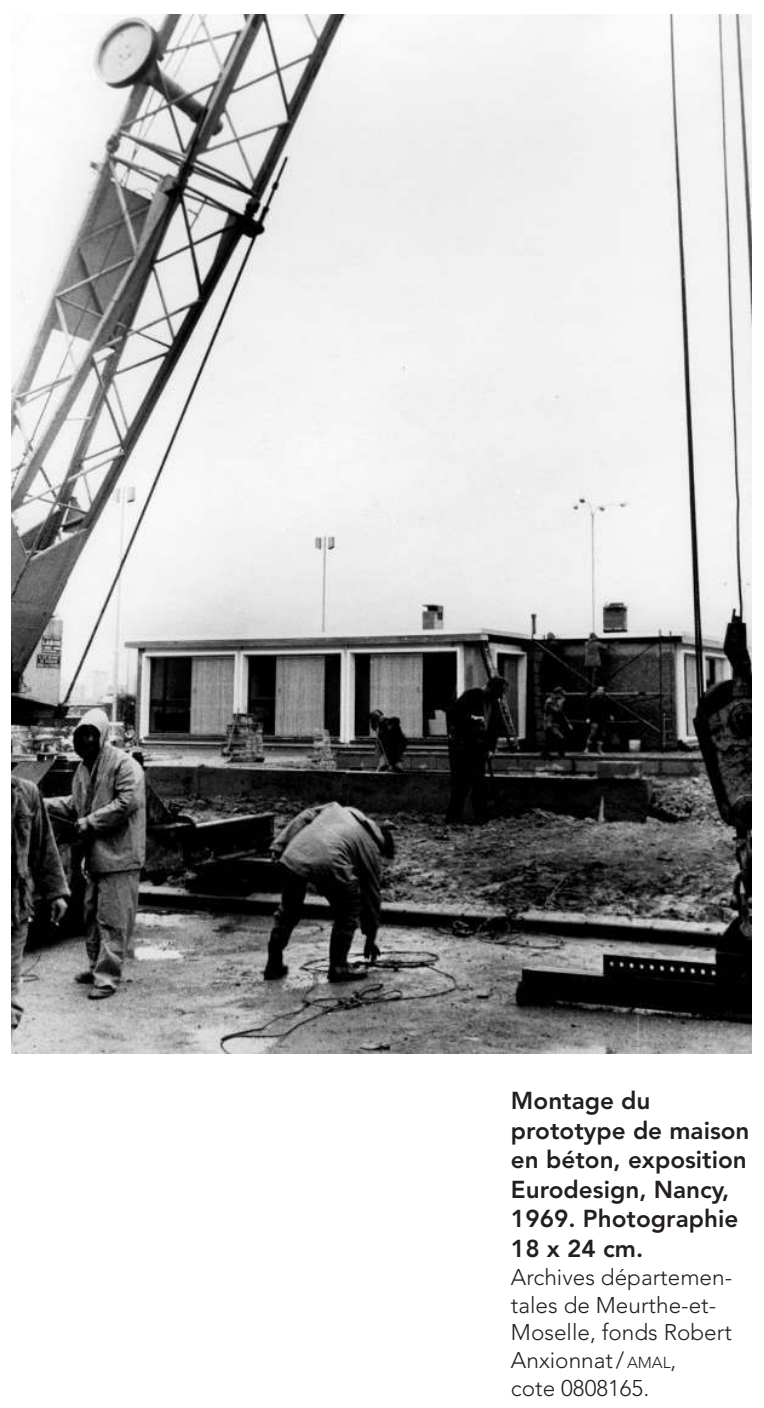

7. CEPRO, La maison modulaire, une architecture nouvelle pour le mieux vivre de la famille, Grenoble, maquette CEPRO, impression Serge Bastien, s.d. (vers 1975), 8 p., archives départementales de Meurthe-et-Moselle, fonds Robert Anxionnat/AMAL, cote 97970009.

8. Robert Anxionnat a été l'architecte d'intérieur, sélectionné sur concours, des bâtiments du Parlement européen de Luxembourg (1965), de la préfecture de
Bobigny (1969-71), de la préfecture de région à Metz (1973-1974), du palais des congrès à Nancy (1977), de la Cour de Justice de I'Union européenne à Strasbourg (1982), etc.

9. "Comment: Eurodesign at Nancy », Design n ${ }^{\circ} 248,1$ août 1969, p. 14-15; "Eurodesign n 2, la maison d'aujourd'hui sera pensée de l'intérieur », Le Journal de Nancy, 13 avril 1969 ; "Eurodesign. Au parc des expositions de Nancy, nouveaux succès du salon-colloque de l'esthétique industrielle ", L'Usine nouvelle $n^{\circ} 37,1969$, p. 44

10. Selon Robert Anxionnat, I'acronyme MAM signifie "Maison modulaire ». II est également possible qu'il ait été choisi pour représenter le nom de l'architecte et de deux entreprises pressenties pour participer à sa fabrication, les entreprises Millet (Château-Salins, Moselle) et Munch (Frouard, Meurthe-et-Moselle). 


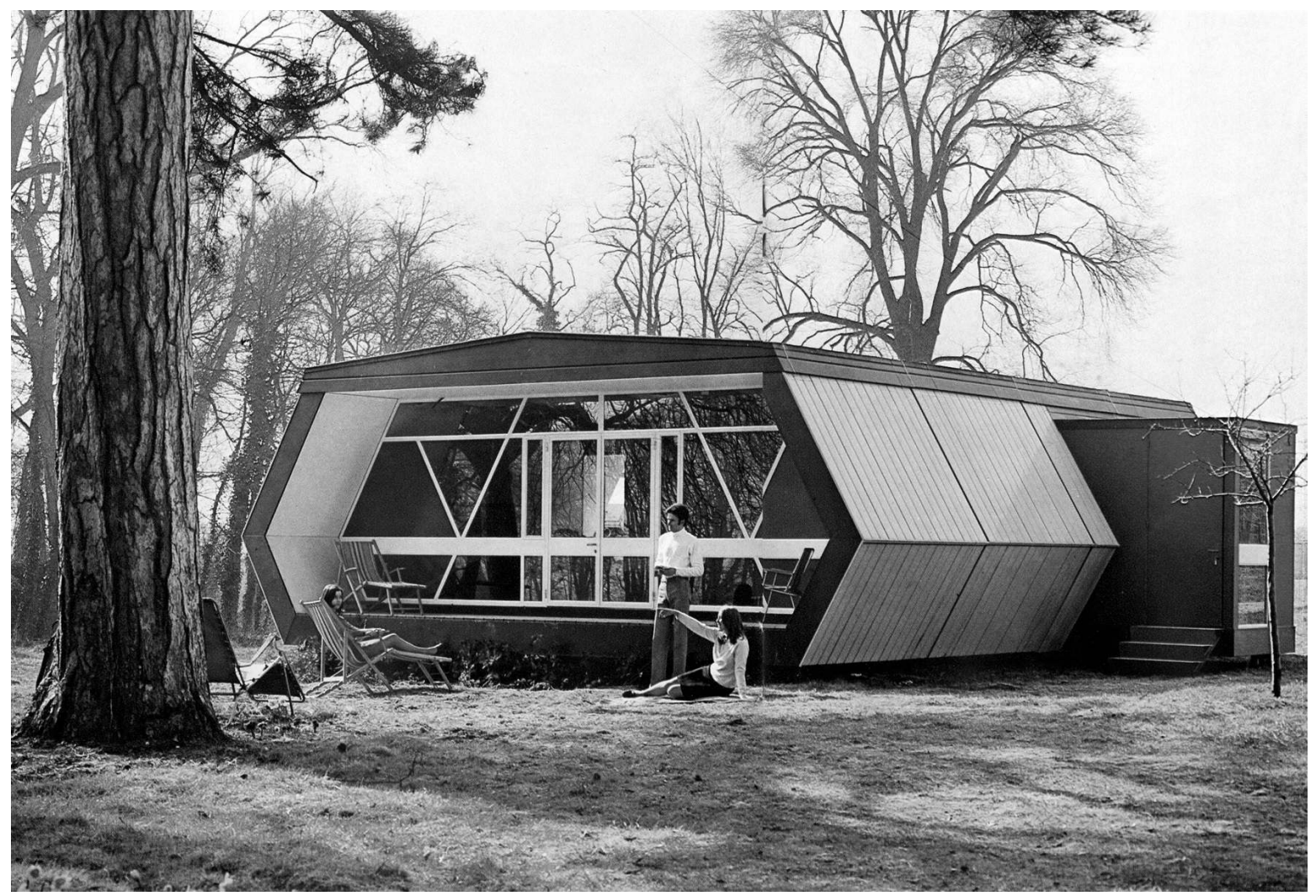

Prototype de chalet MAM, non daté (environ 1970). Photographie, document publicitaire CEPRO, La maison modulaire, une architecture nouvelle pour le mieux vivre de la famille, Grenoble, maquette CEPRO, impression Serge Bastien, s.d. (environ 1975), 8 p. Archives départementales de Meurthe-et-Moselle, fonds Robert Anxionnat/ AMAL, cote 97970009 . 
sera déclinés sous différentes formes et dans de multiples versions, de 1968 jusqu'à la fin des années 1980, donnant lieu à plusieurs tentatives de commercialisation ${ }^{11}$. Au-delà du procédé technique et de la stratégie commerciale, la série est présentée comme une solution urbanistique, comme le montrent les plans d'implantation à échelle territoriale où les systèmes de cellule se développent sans limite ${ }^{12}$. Cette première expérience sera par la suite prolongée par plusieurs autres recherches, et par une réalisation pour le Plan Construction à Blénod-lès-Pont-à-Mousson ${ }^{13}$.

Le choix de consacrer une partie de cette étude aux réalisations de Robert Anxionnat peut porter à questionnement, étant donné que la production de l'architecte dans le domaine des maisons industrialisées est restée quelque peu marginale. Elle ne manque cependant pas d'intérêt, et ce, à plusieurs niveaux. Tout d'abord, cette production est totalement absente de l'historiographie; les sources secondaires la concernant se limitent à une vingtaine d'articles dans des revues dans des années 1960 et 1970. Par ailleurs, la carrière de Robert Anxionnat se situe dans une période temporelle charnière qui verra I'apogée des relations entre architecture et industrie. Pour différentes raisons, cette carrière est empreinte de l'héritage de constructeurs des générations précédentes tels que ceux étudiés dans la première et la seconde partie de la thèse. Enfin, à l'instar de Ferdinand Fillod et de Jean Prouvé, Robert Anxionnat possédait une structure de production qui lui permettait d'envisager de fabriquer lui-même une partie des éléments de construction.

\section{Méthodologies}

Une problématique d'ensemble étant définie pour I'ensemble de la thèse, d'autres problématiques s'y sont superposées pour chacune des trois parties, les histoires de chacun des concepteurs étant très différentes, et apportant chacune une vision distincte de la problématique centrale.

Face à la diversité des sources et des méthodes s'est imposée naturellement la nécessité d'un contrepoids, sous la forme d'une structure commune à chacune des trois parties dans lesquelles se développent les histoires des maisons industrialisées de chaque concepteur. Ainsi, pour chacun d'eux trois, sont tout d'abord étudiées les conditions de conception et de fabrication des maisons industrialisées, puis les méthodes de communication qui ont été employées pour les promouvoir (supports imprimés, événements...), et enfin leur réception par le public spécialiste et non-spécialiste. Ceci permet de conserver une unité à l'ensemble de la thèse, et de constituer une grille d'analyse qui offre la possibilité de comparer et de confronter les projets et les méthodes des concepteurs.

Les méthodes utilisées pour cette recherche ont été très différentes dans les cas de chacun des trois concepteurs étudiés, étant donné qu'elles traitent chacune d'une période bien distincte : selon l'éloignement dans le temps, les sources diffèrent. Par ailleurs, la notoriété du concepteur étudié fait fortement varier les conditions de la recherche.

La première partie, qui retrace le parcours du constructeur Ferdinand Fillod, s'est basée dans un premier temps sur un nombre restreint de sources
11. Curriculum vitae de Robert Anxionnat, fonds Robert Anxionnat/AMAL, ADMM, cote 0808168 .

12. Plans de la maison MAM, archives départementales de Meurthe-et-Moselle, fonds Robert Anxionnat/AMAL, cote 97970009

13. En 1972, Marcel Corre, R.-C. Kruger et Robert Anxionnat sont lauréats du concours «Innovation lorraine », lancé par le Plan Construction du ministère de l'Équipement et du logement, pour la conception et la réalisation de 67 logements HLM à Blénod, prolongée ensuite par la construction de 39 pavillons. Voir, à ce sujet, Joseph Abram et Daniel Gross, Bilan des réalisations expérimentales en matière de technologie nouvelle: Plan Construction 1971-1975, bilan thématique, Paris, Plan Construction, 1983, 185 p. 
secondaires, suite à la découverte de photographies d'un certain nombre de bâtiments, puis la définition du corpus a été possible grâce à des visites in situ, au cours desquelles les bâtiment présélectionnés ont été situés géographiquement et photographiés. Sur la base de ce corpus, différentes consultations d'archives ont permis de progresser dans la recherche, notamment grâce à la découverte de publicités réalisées pour promouvoir les maisons.

La deuxième partie, qui traite des maisons de Jean Prouvé, s'est construite à partir de lectures, de consultations de banques d'archives préalablement ciblées et sélectionnées, et grâce à des entretiens avec des spécialistes.

La troisième partie, qui évoque les maisons réalisées par Robert Anxionnat, a eu pour point de départ la sauvegarde des archives presque complètes de I'agence, qu'il a fallu inventorier et organiser ${ }^{14}$. Ensuite, les bâtiments présélectionnés dans les archives ont fait l'objet de visites, lesquelles ont permis de définir, pour un certain nombre de projets, si les bâtiments avaient été réalisés ou non, s'ils existaient toujours, et dans quelle mesure ils avaient été modifiés. Des édifices uniquement connus grâce à leurs représentations graphiques ont ainsi été découverts et localisés. En parallèle, des entretiens avec les collaborateurs, les clients et les amis de l'architecte ont permis d'apporter des renseignements supplémentaires non négligeables.

Le projet de recherche détaillé, une fois établi, s'est révélé porté par une ligne directrice légèrement différente de celle mise en place lors des premières recherches sur la problématique d'ensemble. Cette ligne tend désormais plutôt à établir un regard critique sur les rêves, les défis et les échecs de différentes conceptions de l'habitat de l'ère industrielle. Au fil des recherches, des édifices inconnus, des maisons dont on ne pouvait pas soupçonner l'existence, ont été découverts ; de fil en aiguille, des liens entre les édifices, les personnages, sont apparus, parfois ténus, parfois dotés de la force d'évidences cachées. Le travail à une échelle détaillée avait permis, pas à pas, de rediriger les grandes lignes du projet de recherche, tout comme le travail à des échelles variées permet la progression du projet architectural.

\section{Le sens de la recherche}

La question de l'utilité d'un travail de recherche est essentielle lors de la production. Si l'on sait que l'objectif de la recherche fondamentale consiste en la production de connaissances, il peut être nécessaire d'imaginer des applications à ces connaissances. La recherche en histoire de l'architecture permet de mieux documenter les travaux d'architectes connus, et de constituer une historiographie relative à des architectes inconnus. Elle identifie des objets remarquables inexplorés (ou presque), et établit à leur sujet une documentation : il s'agit donc d'un travail qui se situe en amont des réflexions sur le patrimoine, et dont l'une des utilités est la production de documents qui participeront à des pensées plus vastes, à une échelle urbaine ou historique plus large, sur la nécessité de conserver ou sur la possibilité de détruire des édifices, ainsi que sur la place qui leur est accordée par notre société.

14. Les archives de l'agence d'architecture de Robert Anxionnat ont été sauvegardées et conservées par Catherine Coley et Jean-Claude Vigato pour l'association AMAL (Archives modernes de l'architecture lorraine). 